\title{
Gastrointestinal modifications and bioavailability of brown seaweed phlorotannins and effects on inflammatory markers
}

\author{
Giulia Corona ${ }^{1,2 *}$, Yang $\mathrm{Ji}^{2}$, Prapaporn Anegboonlap ${ }^{2}$, Sarah Hotchkiss ${ }^{3}$, Chris Gill $^{4}$, Parveen Yaqoob $^{2}$, \\ Jeremy P. E. Spencer ${ }^{2}$ and Ian Rowland ${ }^{2}$ \\ ${ }^{1}$ Health Sciences Research Centre, University of Roehampton, London SW15 4JD, UK \\ ${ }^{2}$ Department of Food and Nutritional Sciences, University of Reading, Reading RG6 6AP, UK \\ ${ }^{3}$ CyberColloids Ltd, Carrigaline Industrial Estate, Carrigaline, County Cork P43 VR72, Republic of Ireland \\ ${ }^{4}$ Northern Ireland Centre for Food \& Health, University of Ulster, Coleraine BT52 1AA, UK \\ (Submitted 22 October 2015 - Final revision received 28 December 2015 - Accepted 7 January 2016 - First published online 16 February 2016)
}

\section{Abstract}

Brown seaweeds such as Ascophyllum nodosum are a rich source of phlorotannins (oligomers and polymers of phloroglucinol units), a class of polyphenols that are unique to Phaeophyceae. At present, there is no information on the bioavailability of seaweed polyphenols and limited evidence on their bioactivity in vivo. Consequently, we investigated the gastrointestinal modifications in vitro of seaweed phlorotannins from A. nodosum and their bioavailability and effect on inflammatory markers in healthy participants. In vitro, some phlorotannin oligomers were identified after digestion and colonic fermentation. In addition, seven metabolites corresponding to in vitro-absorbed metabolites were identified. Urine and plasma samples contained a variety of metabolites attributed to both unconjugated and conjugated metabolites (glucuronides and/or sulphates). In both urine and plasma, the majority of the metabolites were found in samples collected at late time points (6-24h), suggesting colonic metabolism of high-molecular-weight phlorotannins, with three phlorotannin oligomers (hydroxytrifuhalol A, 7-hydroxyeckol, C-O-C dimer of phloroglucinol) identified in urine samples. A significant increase of the cytokine IL- 8 was also observed. Our study shows for the first time that seaweed phlorotannins are metabolised and absorbed, predominantly in the large intestine, and there is a large inter-individual variation in their metabolic profile. Three phlorotannin oligomers present in the capsule are excreted in urine. Our study is the first investigation of the metabolism and bioavailability of seaweed phlorotannins and the role of colonic biotransformation. In addition, IL-8 is a possible target for phlorotannin bioactivity.

Key words: Polyphenols: Phlorotannins: Brown seaweed: Bioavailability: Metabolism: Human subjects

There has been increasing interest in the past few years on the bioactive compounds present in seaweeds ${ }^{(1-3)}$. Traditionally, seaweeds are consumed as a food product in Asian countries and are increasingly used worldwide as ingredients for industrial applications. In Japan, over twenty species of red, green and brown algae (seaweed) are included in meals ${ }^{(4)}$, and daily seaweed consumption per person has remained relatively consistent over the past 40 years, in the range of $1.50-3.65 \mathrm{~kg} /$ person per year, as reported by a range of studies ${ }^{(5-7)}$. Seaweeds are a rich source of polyphenolic compounds ${ }^{(8)}$, and polyphenols extracted from algae ${ }^{(9,10)}$ show some similarities to those found in land plants ${ }^{(9-11)}$. Thus, the main polyphenols found in brown seaweeds are phlorotannins ${ }^{(12-15)}$, a type of phenolic compound only found in brown seaweeds ${ }^{(16)}$. Brown seaweed phlorotannins are oligomers and polymers of phloroglucinol units, and their oligomer and polymer molecular weights can greatly vary, from $126 \mathrm{Da}$ to $650 \mathrm{kDa}^{(3)}$, comprising up to $15 \%$ of the plant dried weight ${ }^{(11)}$. It has been reported that the consumption of brown algae is on average $1.342 \mathrm{~kg} /$ person per year, containing $66.652 \mathrm{~g}$ of phlorotannins/person per year and $183 \mathrm{mg} /$ person per $\mathrm{d}^{(4)}$. Phlorotannins are classified according to the type of linkages between phloroglucinol units into four main groups: eckols (with dibenzodioxin linkages), fucols (with a phenyl linkage), fuhalols and phloroethols (with ether linkages), and fucophloroethols (with ether and phenyl linkages ${ }^{(16)}$. Phlorotannins are being increasingly investigated for their vast array of bioactivities ${ }^{(10,17,18)}$ such as antioxidant $^{(19-24)}$, anti-inflammatory ${ }^{(20,25,26)}$, antibacterial $^{(27,28)}$, anticancer $^{(29-33)}$ and antidiabetic ${ }^{(29,34,35)}$, showing promising potential to further develop seaweed-derived products rich in bioactive components with commercial potential for food and pharma applications ${ }^{(36)}$.

Abbreviations: DM, digestion metabolite; ESI, electrospray ionisation; GI, gastrointestinal; HMW, high-molecular-weight; LPS, lipopolysaccharide; NP-HPLC, normal-phase HPLC; RP-HPLC, reverse-phase HPLC; SPE, seaweed polyphenol extract.

* Corresponding author: Dr G. Corona, fax +44 208392 3610, email giulia.corona@roehampton.ac.uk 
Bioavailability is a critical factor influencing in vivo biological activity of polyphenols and we have reasonable understanding of the bioavailability of polyphenols from fruits and vegetables, and some of the mechanisms by which they exert beneficial effects in vivo have been determined ${ }^{(37)}$. Their ability to act as effective bioactive molecules in vivo is dependent on the extent of their biotransformation ${ }^{(24)}$ and conjugation during absorption from the gastrointestinal (GI) tract, in the liver and finally in cells $^{(37)}$. Consequently, consideration must be given to the way polyphenols are absorbed and metabolised during GI digestion and colonic fermentation, and how this may have an impact on bioactivity $^{(38)}$. It is noteworthy that there is no information on the bioavailability of seaweed phlorotannins, and this is a limitation to understanding their bioactivity and mechanism of action in vivo. In the absence of specific data regarding phlorotannin absorption and bioavailability, it is useful to consider the absorption and metabolism of other polyphenols as a guide ${ }^{(37)}$. In general, after ingestion of a polyphenol-rich diet, their protective effects in vivo are determined by measuring a range of suitable biomarkers, and they correlate with the absorption of polyphenols from the gut and their circulation/excretion $^{(38)}$. Polyphenols can be extensively conjugated to form glucuronide, sulphate and methyl group in the gut mucosa and inner tissues ${ }^{(37,39)}$, and absorption occurs in the small intestine ${ }^{(37)}$. Polyphenols unabsorbed in the upper GI tract or re-excreted in the bile are extensively metabolised by colonic microflora into a wide range of low-molecular-weight phenolic acids ${ }^{(40)}$. The aim of this study was to elucidate the GI modifications of seaweed phlorotannins, and the effects on their metabolism and bioavailability. A food-grade seaweed polyphenol extract (SPE) rich in phlorotannins (from the brown seaweed Ascophyllum nodosum) was subjected to in vitro GI digestion and fermentation to examine the GI modifications occurring in the upper and lower GI tract. Furthermore, the absorption and metabolism of polyphenols in healthy subjects was investigated, after oral ingestion of a SPE capsule containing $101.89 \mathrm{mg}$ of polyphenols. This amount represents an intake lower than the average daily intake of seaweed polyphenols in the Asian diet, and it is not expected to exert any cytotoxic effect ${ }^{(41)}$. The impact of absorption and GI modifications on phlorotannins anti-inflammatory potential is explored.

\section{Methods}

\section{Seaweed material}

Fresh A. nodosum was supplied by The Hebridean Seaweed Company, Isle of Lewis, Scotland in March 2011. The seaweed biomass was harvested by hand, cleaned and then shipped refrigerated to the processing facility in France where it was immediately chopped and frozen

\section{Preparation of food-grade seaweed polyphenol extract and capsule}

A novel SPE from A. nodosum was produced by CEVA (France) using a solvent-based extraction system that was specifically developed for this study and for use with either fresh or frozen A. nodosum. The solvent used was a 60:40 ethanol-water mixture, which allowed for the water content of the seaweed itself. The extraction was carried out over $5 \mathrm{~h}$ using constant stirring and at all times protected from light. A solventseaweed ratio of 3:1 was used. The mixture was filtered to remove the supernatant, and subsequently the alcohol was removed using a rotary evaporator. A hydrometer was used to check that all of the alcohol had been removed. The final extract was recovered by centrifugation and further filtration before freeze-drying.

Approximately half of the produced extract (basic extract) was then fractionated using tangential flow ultrafiltration to produce further extracts of varying molecular-weight range and with varying polyphenol content. A blended SPE was formulated (Table 1) using $175 \mathrm{mg}$ of basic extract and $50 \mathrm{mg}$ of high-molecular-weight (HMW) fraction ( $>10 \mathrm{kDa}$ cut-off) for use in the current study. Maltodextrin $(175 \mathrm{mg})$ was added to the capsule formulation as an excipient. This was done in order to maximise the polyphenol content $(>100 \mathrm{mg} / \mathrm{d})$ but also to minimise the level of I to within accepted regulatory guidelines $(<500 \mu \mathrm{g} / \mathrm{d})$. Blending was carried out at the food-grade CEVA facilities in France. Doses of $400 \mathrm{mg}$ of the SPE Ascophyllum blend were packed into white, opaque, vegetarian capsules by Irish Seaweeds, Belfast, UK and used for the clinical study. The food-grade seaweed capsule was characterised by normal-phase (NP)-HPLC (NP-HPLC) and liquid chromatography (LC)-MS analysis. Phlorotannins were quantified using the Folin-Ciocalteu Method ${ }^{(42)}$ using phloroglucinol as the standard $^{(8)}$.

\section{Simulated gastrointestinal digestion and fermentation}

The GI digestion procedure was adapted from Mills et al. ${ }^{(43)}$ (2008) and McDougall et al. ${ }^{(44)}$ (2005). This method consists of two sequential stages: gastric digestion and small intestinal digestion followed by dialysis. A measure of $10 \mathrm{~g}$ of SPE was

Table 1. Key components of polyphenol-rich basic extract, highmolecular-weight (HMW) fraction and blend (capsule) showing crucial concentrations of polyphenols and iodine

\begin{tabular}{lccc}
\hline & $\begin{array}{c}\text { Basic extract } \\
(\mathrm{mg} / 175 \mathrm{mg} \text { of } \\
\text { extract) }\end{array}$ & $\begin{array}{c}\text { HMW fraction } \\
(\mathrm{mg} / 50 \mathrm{mg} \text { of } \\
\text { extract) }\end{array}$ & $\begin{array}{c}\text { Blend } \\
\text { (capsule) } \\
\text { (mg/400 mg } \\
\text { capsule) }\end{array}$ \\
\hline Extract components & 58.74 & 43.15 & 101.89 \\
Polyphenols & 0.46 & 0.02 & 0.48 \\
I & & & 175 \\
Maltodextrin* & 37.77 & 1.22 & 38.99 \\
Minerals & $<0.001$ & 0.004 & 0.004 \\
Fucoxanthin & 10.24 & 1.64 & 11.88 \\
Laminarin as glucose & $<0.001$ & 0.23 & 0.23 \\
Fucoidan as fucose & 28.03 & 5.53 & 33.56 \\
Mannitol & $<0.001$ & $<0.001$ & $<0.001$ \\
Inorganic arsenic & $<$ LD & $<$ LD & $<$ LD \\
Cd (LD 0.15 mg/kg) & $<$ LD & $<$ LD & $<$ LD \\
Hg (LD 0.016 mg/kg) & $<$ LD & $<$ LD & $<$ LD \\
Pb (LD 1.1 mg/kg) & $<$ LD & $<$ LD & $<$ LD \\
Sn (LD 1.7 mg/kg) & & & \\
\hline
\end{tabular}

LD, limit of detection.

* Maltodextrin was added to the capsule formulation as an excipient. 
dissolved in $30 \mathrm{ml}$ of acidified water $(\mathrm{pH}=2)$, and pepsin $\left(320 \mathrm{U} / \mathrm{ml}\right.$ ) was added. Samples were incubated at $37^{\circ} \mathrm{C}$ for $2 \mathrm{~h}$ on a shaker covered with foil to protect them from light. Aliquots of $5 \mathrm{ml}(\mathrm{G})$ were removed. The $\mathrm{pH}$ was adjusted to 7.5 by adding a few drops of $6 \mathrm{~m}-\mathrm{NaOH}$, and pancreatin $(4 \mathrm{mg} / \mathrm{ml})$ and bile extracts $(25 \mathrm{mg} / \mathrm{ml})$ were added. The samples were incubated at $37^{\circ} \mathrm{C}$ for $2 \mathrm{~h}$ on a shaker. Aliquots of $5 \mathrm{ml}$ (SI) were removed. Samples were transferred into the dialysis tubing (100-500 Da, cutoff, $1.8 \mathrm{ml} / \mathrm{cm}$, Spectra/Por; Biotech) and dialysed overnight at $4{ }^{\circ} \mathrm{C}$ against water ( 4 litres) to remove low-molecular-weight digests. Aliquots of $5 \mathrm{ml}$ of dialysis solution (D1) were removed. The dialysis fluid was changed and dialysis was continued for an additional $2 \mathrm{~h}$. Aliquots of $5 \mathrm{ml}$ of second dialysis solution (D2) were removed. Samples (SI+D) were freeze-dried and subjected to colonic fermentation (batch culture): the method was adapted from Tzounis et al. ${ }^{(45)}$ Batch-culture fermentation vessels ( $300 \mathrm{ml}$; one vessel per treatment) were autoclaved and filled with $135 \mathrm{ml}$ of sterilised basal medium. Medium was stirred and gassed overnight with $\mathrm{O}_{2}$-free $\mathrm{N}_{2}$. Before the addition of SI+ D-digested extracts equivalent to $1.5 \mathrm{~g}$ of undigested extracts, the temperature inside the vessels was set to $37^{\circ} \mathrm{C}$ by a circulating water bath, and the $\mathrm{pH}$ was controlled at 6.8 by an Electrolab $\mathrm{pH}$ controller (Electrolab UK), in order to mimic conditions in the distal region of the human large intestine (anaerobic; $37^{\circ} \mathrm{C}$; $\mathrm{pH}$ 6.8). Vessels were inoculated with $15 \mathrm{ml}$ of faecal slurry $(1: 10, \mathrm{w} / \mathrm{v})$, and batch cultures were run for $24 \mathrm{~h}$. Samples measuring $7 \mathrm{ml}$ were collected at five time points (0, 2, 4, 8 and $24 \mathrm{~h}$ ), centrifuged at $13000 \mathrm{rpm}$ at $4^{\circ} \mathrm{C}$ for $10 \mathrm{~min}$ and the supernatants were kept. All the samples collected during the digestion and fermentation procedures were stored at $-80^{\circ} \mathrm{C}$ until LC-MS analysis.

\section{Study design}

This study was conducted according to the guidelines laid down in the Declaration of Helsinki, and all procedures involving human subjects/patients were approved by the University of Reading Ethics Committee before initiation of the study. Written informed consent was obtained from all participants. Exclusion criteria for subjects were as follows: smokers, $\mathrm{BMI}<18$ or $>30 \mathrm{~kg} / \mathrm{m}^{2}$, abnormal liver function and haematology, alcohol intake of $>21 \mathrm{U} /$ week, GI disease or chronic GI disorders, consumption of antibiotics in previous 3 months before study and women who were pregnant or intending to become pregnant. Potentially suitable participants underwent a screening process, and individuals with blood pressure $>150 / 90 \mathrm{mmHg}$; $\mathrm{Hb}>125 \mathrm{~g} / \mathrm{l}$ for men and >110 g/l for women, $\gamma$-glutamyl transferase $>1.3 \mathrm{mkat} / \mathrm{l}$ or cholesterol $>6.5 \mathrm{mmol} / \mathrm{l}$ were excluded from the study. In total, twenty-four volunteers were recruited: twelve female volunteers (six aged 18-30 years and six aged 30-65 years) and twelve male volunteers (six aged 18-30 years and six aged 30-65 years). Participants were asked to follow a low phenolic diet for $1 \mathrm{~d}$ before the study day (devoid of tea, coffee, fruit, vegetables, alcoholic beverages, cocoa, whole-grain and seaweed-containing products). On the day of the study, the subjects were cannulated and a baseline blood sample was taken. Participants were asked to consume one SPE capsule ( $400 \mathrm{mg}$ ) containing $101.89 \mathrm{mg}$ of polyphenols. Blood samples were collected at 0, 1, 2, 3, 4, 6, 8 and $24 \mathrm{~h}$ after ingestion of the SPE capsule, and urine samples were collected at baseline, 0-8 and 8-24 h after the ingestion. During the day, participants were provided with a lunch and dinner of low phenolic content. The study is registered at clinicaltrials.gov (study ID: NCT02496806).

\section{Sample collection and storage}

One aliquot of blood was collected in heparin tubes and cultured immediately (whole blood culture for cytokine analysis). One aliquot of blood was collected in EDTA tubes and centrifuged at $3000 \mathrm{rpm}$ for $15 \mathrm{~min}$ at $4^{\circ} \mathrm{C}$. The plasma was separated and $1 \mathrm{mg} / \mathrm{ml}$ ascorbic acid was added as preservative. Aliquots were stored at $-80^{\circ} \mathrm{C}$ until analysis. Total volume of collected urine was recorded, and aliquots were stored at $-80^{\circ} \mathrm{C}$ until analysis.

\section{Plasma sample processing for metabolite analysis}

Plasma samples were prepared by following a procedure similar to the one described by Ottaviani et al. ${ }^{(46)}$. A volume of $10 \mu \mathrm{l}$ of internal standard solution (resorcinol $200 \mu \mathrm{g} / \mathrm{ml}$ ) was added to $450 \mu \mathrm{l}$ of plasma, and then $50 \mu \mathrm{l}$ of $1.2 \mathrm{M}$-acetic acid was added and samples were mixed. Samples were analysed with and without enzymatic treatment $\left(37^{\circ} \mathrm{C}, 40 \mathrm{~min}\right)$ in the presence of $1500 \mathrm{IU}$ of $\beta$-glucuronidase and $50 \mathrm{IU}$ of sulfatases from Helix pomatia (type H-1). A volume of $1 \mathrm{ml}$ of $100 \%$ methanol acidified with $0.5 \%$ acetic acid was added, and samples were centrifuged for $15 \mathrm{~min}$ at $16100 \mathrm{~g}$ at $4^{\circ} \mathrm{C}$ and supernatants were collected. This step was repeated three times (last time with $50 \%$ methanol acidified with $0.5 \%$ acetic acid), and the supernatants were dried using a SpeedVac. The pellets were dissolved with $125 \mu \mathrm{l}$ of mobile phase and transferred to vials for reverse-phase (RP)-HPLC (RP-HPLC) analysis.

\section{Urine sample processing for metabolite analysis}

Urine samples were prepared according to a procedure similar to the one described by Ottaviani et al. ${ }^{(46)}$. Briefly, $10 \mu \mathrm{l}$ of internal standard solution (resorcinol $200 \mu \mathrm{g} / \mathrm{ml}$ ) was added to $250 \mu \mathrm{l}$ of urine. Samples were analysed with and without enzymatic treatment $\left(37^{\circ} \mathrm{C}, 40 \mathrm{~min}\right)$, in the presence of $1500 \mathrm{IU}$ of $\beta$-glucuronidase and $50 \mathrm{IU}$ of sulfatases from $H$. pomatia (type $\mathrm{H}-1$ ). A volume of $1 \mathrm{ml}$ of $100 \%$ methanol acidified with $0.5 \%$ acetic acid was added, samples were mixed and centrifuged for $15 \mathrm{~min}$ at $16100 \mathrm{~g}$ at $4^{\circ} \mathrm{C}$, and supernatants were transferred to a new tube and dried on a SpeedVac. Dried samples were re-suspended on $125 \mu \mathrm{l}$ of mobile phase, completely dissolved, centrifuged and transferred to vials for RP-HPLC analysis and LC-MS analysis.

\section{Normal-phase HPLC analysis}

The phlorotannins in the food-grade SPE used to produce the capsule were analysed by NP-HPLC analysis ${ }^{(8)}$ using an HPLC 1100 series equipped with LiChrospher Si60-5 column ( $250 \mathrm{~mm} \times 4.0 \mathrm{~mm}$ ID, $5 \mu \mathrm{m}$ particle size from Hichrom (LISP605-250AF)), fitted with a guard column LiChrospher Si60-5 from HICHROM (LISP60-5-10C5). The mobile phase contained A: $82 \%$ 
dichloromethane $+2 \%$ methanol $+2 \%$ acetic acid in water and B: $96 \%$ methanol $+2 \%$ acetic acid in water, and it was pumped through the column at a rate of $1 \mathrm{ml} / \mathrm{min}$. A volume of $10 \mu \mathrm{l}$ of samples was injected and analysed by the gradient programme, which was ( $\mathrm{min} / \% \mathrm{~B})$ as follows: 0/0, 30/17·6, 45/30·7, 50/87·8, $60 / 87 \cdot 8,80 / 0,105 / 0$ for detection of all compounds. The compounds were detected at a wavelength of $268 \mathrm{~nm}$. All data were analysed by the ChemStation software. The phloroglucinol standard was injected at $0 \cdot 1-100 \mu \mathrm{g} / \mathrm{ml}$, and phlorotannins in the capsules were analysed as phloroglucinol equivalents.

\section{Reverse-phase HPLC analysis}

The analysis of plasma and urine samples was carried out with a Hewlett-Packard 1100 series liquid chromatograph (HewlettPackard), as previously described ${ }^{(47)}$. Samples were analysed by RP-HPLC using a Nova-Pak C18 column $(4 \cdot 6 \times 250 \mathrm{~mm})$ with $4 \mu \mathrm{m}$ particle size. The temperature of the column was maintained at $30^{\circ} \mathrm{C}$. The mobile phases consisted of a mixture of $5 \%$ aqueous methanol in $0 \cdot 1 \%$ hydrochloric acid $5 \mathrm{~m}$ (A) and a mixture of aqueous acetonitrile 50 in $0.1 \%$ hydrochloric acid 5 м (B), and they were pumped through the column at a rate of $0.7 \mathrm{ml} / \mathrm{min}$. The following gradient system was used ( $\mathrm{min} / \% \mathrm{~B})$ : $0 / 5,5 / 5,40 / 50,55 / 100,59 \cdot 9 / 100,60 / 5$, with 10 min post-run for both compound and metabolite detections. The eluent was monitored by photodiode array detection at $280 \mathrm{~nm}$ and spectra of products obtained over the $200-600 \mathrm{~nm}$ range. Peaks were characterised by their retention time and spectra characteristics. A calibration curve of phloroglucinol was constructed using authentic standards $(0 \cdot 1-100 \mu \mathrm{g} / \mathrm{ml})$, and in each case they were found to be linear with correlation coefficients of 0.995 . Metabolites were quantified as phloroglucinol equivalents.

\section{Liquid chromatography-MS analysis}

LC-MS analysis was conducted to analyse the food-grade seaweed capsule, the urine samples and the digested materials, and it was carried out in the negative ion mode using LC-MS/MS utilising electrospray ionisation (ESI), as previously described $^{(48)}$. Characterisation was achieved using LC-MS/MS utilising ESI. This consisted of an Agilent 1200 HPLC system equipped with a binary pump, degasser, auto-sampler, thermostat, column heater, photodiode array detector and an Agilent 1100 series LC/MSD Mass Trap Spectrometer (Agilent Technologies UK). Separation of samples was achieved using a Zorbax SB C18 column $(2 \cdot 1 \times 100 \mathrm{~mm} ; 1.8 \mu \mathrm{m})$ (Agilent), and HPLC conditions were as follows: injection volume, $1 \mu \mathrm{l}$; column temperature, $25^{\circ} \mathrm{C}$; binary mobile system, (A) $0.1 \%$ aqueous formic acid and (B) $0.1 \%$ of formic acid in acetonitrile; and flow rate, $0.2 \mathrm{ml} / \mathrm{min}$. A series of linear gradients were used for separation $(\mathrm{min} / \% \mathrm{~B})$ : $0 / 10,3 / 10,15 / 40,40 / 70,50 / 70,65 / 10$. MS was performed in the negative ion mode (scan range, $m / z$ 100-1000 Da; source temperature, $350^{\circ} \mathrm{C}$ ). All solvents used were of LC-MS grade.

\section{Cytokine production}

Blood samples collected during the clinical intervention (baseline, 1, 2, 4, 6 and $8 \mathrm{~h}$ ) into heparin tubes were immediately cultured as follows: $500-\mu \mathrm{l}$ blood aliquots were mixed with
$500 \mu \mathrm{l}$ of Roswell Park Memorial Institute (RPMI) medium containing antibiotics on a twenty-four-well plate, and lipopolysaccharide (LPS) $(1 \mu \mathrm{g} / \mathrm{ml})$ or vehicle (control group) was added before incubation at $37^{\circ} \mathrm{C}$ for $24 \mathrm{~h}$. At the end of the culture period, samples were centrifuged at $2000 \boldsymbol{g}$ for $5 \mathrm{~min}$, and the supernatants were collected and kept at $-20^{\circ} \mathrm{C}$ until analysis for inflammatory cytokine levels. The supernatants were collected and stored at $-20^{\circ} \mathrm{C}$. Cytokines (IL- $1 \beta$, IL-6, IL-8, IL-10 and TNF- $\alpha$ ) in the supernatants were measured with Luminex xMAP Technology using commercially available Fluorokine MAP kits (R\&D systems), and data were analysed on the xPONENT software. Final data are presented as the difference between LPS-treated and unstimulated control.

\section{Statistical analysis}

The statistical evaluation of the results was performed by oneway ANOVA, followed by a Bonferroni post hoc $t$ test using GraphPad InStat version 5 (GraphPad Software). Significant changes are indicated as $P<0 \cdot 05$.

\section{Results}

\section{Seaweed polyphenol extract characterisation}

The chromatogram (Fig. 1) illustrates the trace obtained by NP-HPLC with diode array detection after injecting a water solution of the SPE. The chromatogram shows a number of peaks (20-70 min) representing different HMW phlorotannins, the characteristic phenolics in brown seaweeds. Longer phlorotannin polymers, which consisted of more hydroxy groups, resulted in tighter attachment to the column material. Consequently, shorter compounds were released earlier than longer compounds. Owing to a lack of phlorotannin standards and the complexity of the oligomeric and polymeric forms, the calibration curve of phloroglucinol was used to quantify all the phlorotannins contained in the SPE as phloroglucinol equivalents. The SPE comprised a wide range of molecular weights of phloroglucinol derivatives with a total phlorotannin concentration of $312 \mu \mathrm{g} / \mathrm{mg}$ quantified as phloroglucinol equivalents. Further characterisation of the SPE was achieved with LC-MS/MS (Fig. 2) using ESI. The data were collected in a non-targeted manner, by acquiring full spectrum data in negative ion mode from $\mathrm{m} / z 100$ to 1000 . The data were then analysed by searching for the theoretical mono-isotopic masses corresponding to possible phlorotannin oligomers and the

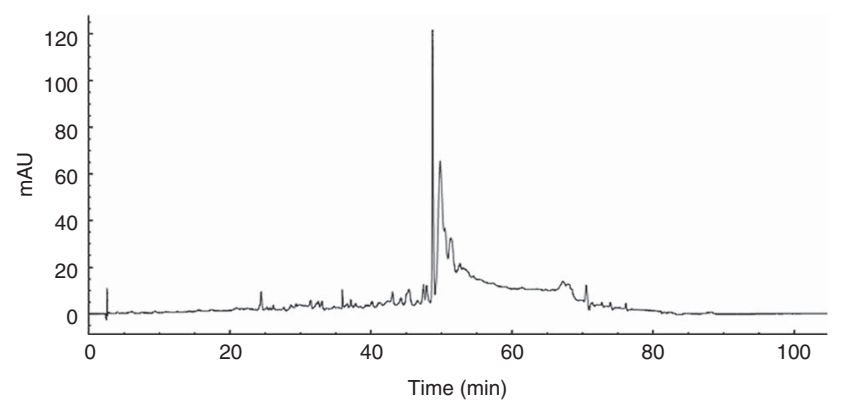

Fig. 1. Chromatographic separation of phlorotannins contained in the seaweed extract by normal-phase HPLC with diode array detection $(268 \mathrm{~nm})$. 
(a)

(m)
(b)

Fig. 2. Characterisation of phlorotannins in the seaweed extract. (a) Structures of phlorotannins identified in the seaweed extract. (b) Phlorotannins in the seaweed extracts identified by liquid chromatography (LC)-MS analysis in negative ion mode.

presence of ions (1-6), which could correspond to phlorotannins (Fig. 2). The ion 1 with $[\mathrm{M}-\mathrm{H}]-$ at $m / z 405$ corresponded to the trimer hydroxytrifuhalol A, whereas the MS2 fragment -387 corresponded to the loss of one molecule of water (-18), a characteristic pattern of phlorotannin fragmentation. Compound 2 ([M-H]- at $m / z$ 497) was considered to be a phlorotannin tetramer composed of 4 phloroglucinol units, such as tetrafucol or fucodiphlorethol, and also in this case the fragment -479 corresponds to the loss of a molecule of water $(-18)$, whereas the fragment -353 corresponds to the loss of water $(-18)$ and a phloroglucinol unit (-126), in accordance with analytical profiles recently described in positive ion mode by Wang et al. ${ }^{(49)}$ and by Ferreres et $a l .{ }^{(50)}$. The ion 3 has a $[\mathrm{M}-\mathrm{H}]-$ at $\mathrm{m} / z 247$ corresponding to a C-O-C dimer of phloroglucinol, as previously indicated by Nwosu et $a l^{(29)}$. The ion 4 (387) corresponds to the trimer 7-hydroxyeckol, and we observe the presence of a fragment at -369 deriving from the loss of one molecule of water. Isomers 5 and 6 with [M-H]- at $m / z 249$ were also observed, which can correspond to the dimers diphlorethol and difucol.

\section{In vitro digestion and characterisation}

Because of the lack of commercially available standards for phlorotannins and the complexity of the oligomers and polymers in the extract, the analysis of phlorotannins and their metabolites is challenging and requires a combination of approaches. Similarly to other polyphenol classes, phlorotannins may undergo extensive modification by phase I and phase II enzymes and the colonic microbiota during their transit through the GI tract ${ }^{(37)}$, and the implication of such metabolic modifications on the bioactivity of phlorotannins has not been investigated yet. Consequently, we subjected the SPE to in vitro digestive and fermentative processes. An in vitro gastric and ileal digestion and colon microbial fermentation of the SPE was initially conducted, followed by dialysis to simulate absorption into the circulation. The MS spectra and fragmentations of the compounds detected in the samples were studied (Fig. 3). After in vitro digestion and fermentation procedures, the samples were analysed by LC-MS/MS using ESI in negative ion mode, as previously described, searching for the theoretical mono-isotopic masses corresponding to the lowmolecular-weight phlorotannins previously identified in the capsule (Fig. 2). We were able to identify molecular ions and fragments corresponding to hydroxytrifuhalol A (405), the C-O-C dimer of phloroglucinol (247), the dimer diphlorethol/ difucol (249) and 7-hydroxyeckol (387), also found after colonic fermentation. In addition, in digested and fermented samples subjected to dialysis to mimic absorption into the circulation, we reported the presence of seven compounds (digestion metabolite (DM)1-DM7) corresponding to in vitro-absorbed metabolites.

\section{Plasma and urine analysis}

The food-grade SPE was given to healthy subjects (Fig. 4) in the form of a capsule ( $400 \mathrm{mg}$ ) containing $101.89 \mathrm{mg}$ of 
(a)
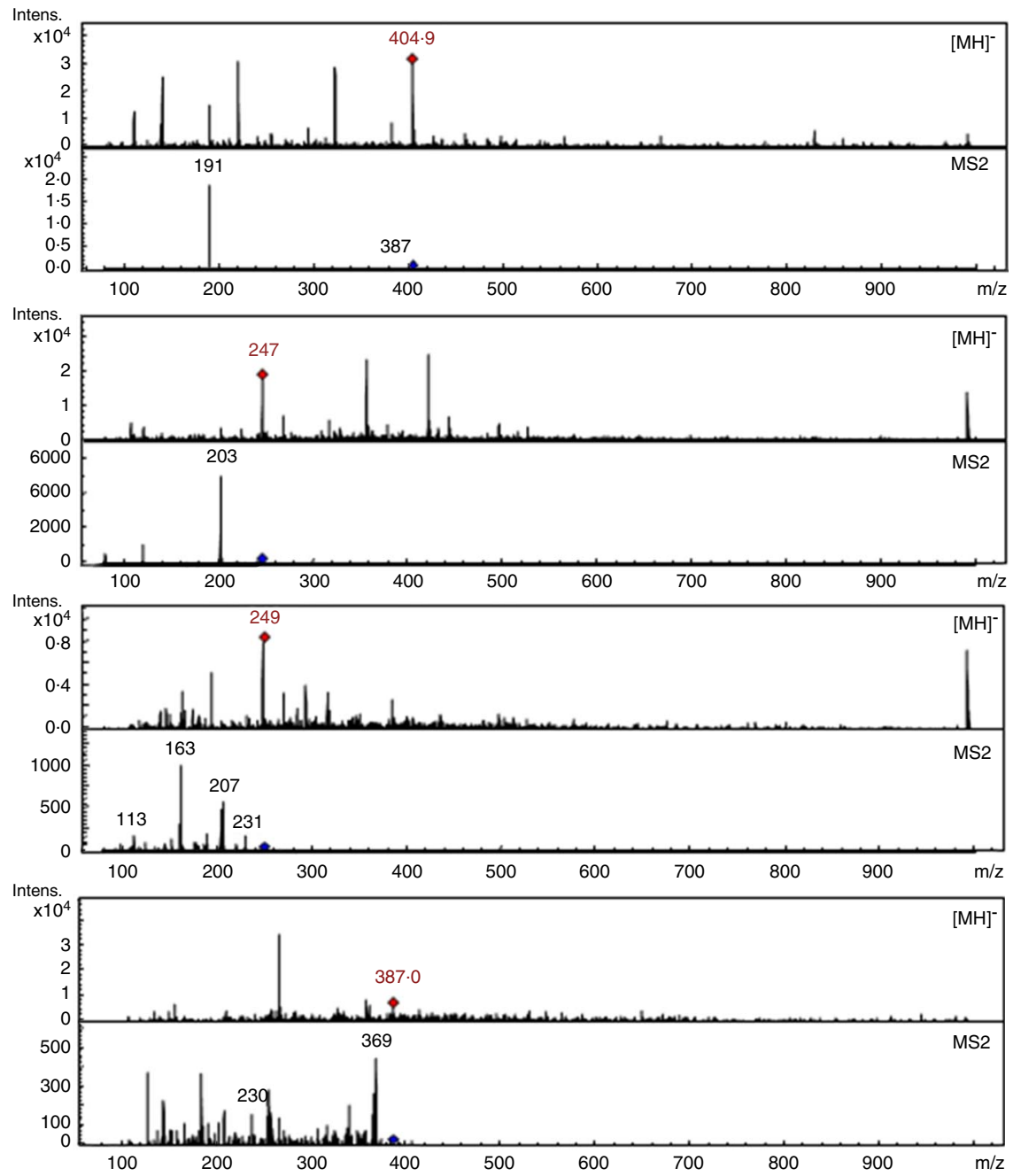

(b)

LC-MS analysis in negative ion mode of the in vitro digested seaweed extracts

\begin{tabular}{|c|c|c|c|c|c|c|c|}
\hline Metaboite & $\begin{array}{c}\mathrm{RT} \\
(\mathrm{min})\end{array}$ & {$[\mathrm{M}-\mathrm{H}]-(\mathrm{m} / \mathrm{z})$} & & \multicolumn{2}{|c|}{$\operatorname{MS} 2(m / z)$} & & Tentative identification \\
\hline \multicolumn{8}{|c|}{ Seaweed extract after small intestinal digestion } \\
\hline SIM1 & $2 \cdot 70$ & 405 & 387 & \multirow{2}{*}{\multicolumn{2}{|c|}{191}} & & \multirow{2}{*}{$\begin{array}{c}\text { Hydroxytrifuhalol A } \\
\text { C-O-C dimer of phloroglucinol }\end{array}$} \\
\hline SIM2 & $5 \cdot 00$ & 247 & 203 & & & & \\
\hline SIM3 & $5 \cdot 70$ & 249 & 231 & 207 & 163 & 113 & Diphlorethol/difucol \\
\hline SIM4 & $9 \cdot 30$ & 387 & 369 & \multicolumn{2}{|l|}{230} & & 7-Hydroxyeckol \\
\hline \multicolumn{8}{|c|}{ Seaweed extract after colonic fermentation } \\
\hline FM1 & $9 \cdot 40$ & 387 & 369 & \multicolumn{2}{|l|}{230} & \multicolumn{2}{|r|}{ 7-Hydroxyeckol } \\
\hline \multicolumn{8}{|c|}{ Dialysed seaweed extract (after digestion and fermentation). } \\
\hline DM1 & $1 \cdot 50$ & 377 & 311 & 243 & 175 & & Unknown \\
\hline DM2 & $1 \cdot 60$ & 277 & 211 & 151 & 93 & & Unknown \\
\hline DM3 & $1 \cdot 65$ & 249 & 181 & 113 & & & Unknown \\
\hline DM4 & $1 \cdot 70$ & 289 & 261 & 221 & 175 & & Unknown \\
\hline DM5 & $1 \cdot 75$ & 317 & 249 & 225 & 181 & 113 & Unknown \\
\hline DM6 & $1 \cdot 80$ & 327 & 269 & 211 & 93 & & Unknown \\
\hline DM7 & 1.85 & 377 & 341 & 281 & 221 & 179 & Unknown \\
\hline
\end{tabular}

Fig. 3. Liquid chromatography (LC)-MS analysis in negative ion mode of the seaweed extract subjected to in vitro gastrointestinal digestion, colonic fermentation and dialysis to mimic absorption. (a) LC-MS spectra and fragmentation of in vitro-digested materials. (b) Summary of LC-MS analysis of the in vitro-digested materials. DM, digestion metabolite. SIM, small intestinal metabolite; FM, fermentation metabolite. 


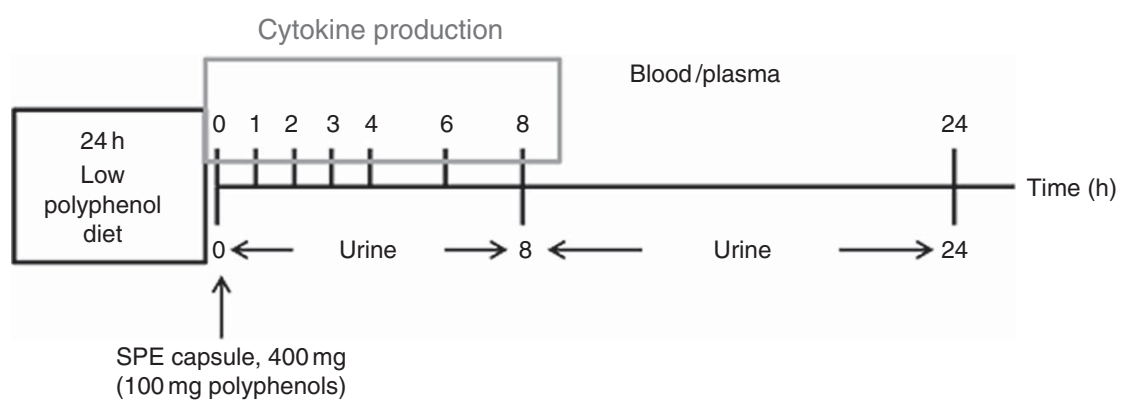

Fig. 4. Schematic illustration of the clinical intervention setup. SPE, seaweed polyphenol extract.

polyphenols, and blood and urine samples were analysed for phlorotannin metabolites. HPLC-diode array detector (DAD) analysis of the plasma (Fig. 5) and urine (Fig. 6) samples with and without glucuronidase/sulfatase treatment showed the presence of a variety of metabolites absent in the baselines (before the seaweed capsule ingestion) in samples from fifteen volunteers out of twenty-four. In plasma, the total level of phlorotannins and their metabolites ranges from 0.011 to $7.757 \mu \mathrm{g} / \mathrm{ml}$, and in urine 0.15 to $33.52 \mu \mathrm{g} / \mathrm{ml}$ are excreted. Some metabolite peaks were present in samples with and without enzymatic treatment, and therefore could be assigned to unconjugated metabolites. Some other metabolite peaks were present only in samples without enzymatic treatment or were only appearing in samples enzymatically treated, and were attributed to conjugated forms (glucuronides and/or sulphates) and their enzymatically released unconjugated forms. In urine, some metabolites were found in samples collected at 0$8 \mathrm{~h}$ after capsule ingestion, but the majority of the metabolites were found in samples collected at $8-24 \mathrm{~h}$. Some metabolites, such as urine metabolite (UM)6 and UM7, show similar UV spectra characteristics and might therefore be structurally related (Fig. 6). In plasma (Fig. 5), some metabolites were found in samples collected at 2, 3 and $4 \mathrm{~h}$ after capsule ingestion, but the majority of the metabolites were found in samples collected at later time points $(6-24 \mathrm{~h})$. This could be the result of poor absorption of the HMW phlorotannins in the upper GI tract, resulting in them reaching the colon and undergoing fermentation to lower-molecular-weight derivatives by the colonic microbiota. In addition, urine samples were subjected to LC-MS/MS (Fig. 7) using ESI, as previously described, searching for the theoretical mono-isotopic masses corresponding to the low-molecular-weight phlorotannins previously identified in the capsule (Fig. 2). We were able to identify molecular ions and fragments corresponding to hydroxytrifuhalol A, 7-hydroxyeckol and the C-O-C dimer of phloroglucinol, which corresponded to the HPLC metabolite UM3. In addition, we reported the presence of two ions (289 and 377) corresponding to metabolites that we characterised in samples from SPE subjected to in vitro GI digestion and fermentation (DM4 and DM7, Fig. 3), as previously detailed.

\section{Ex vivo cytokine production}

The ex vivo production of cytokines (IL-1 $\beta$, IL-6, IL-8, IL-10 and TNF- $\alpha$ ) relative to baseline levels in cultured blood collected a various time points $(0,1,2,3,4,6$ and $8 \mathrm{~h})$ during the intervention study (LPS treated - unstimulated controls) is reported in Fig 8. The amounts of TNF- $\alpha$ and IL-10 remained quite stable over time, as well as the amount of all cytokines at 1 and $2 \mathrm{~h}$. IL- 6 levels were observed to decrease at later time points (4-8h) without reaching statistical significance $(P>0 \cdot 05)$. The levels of both IL-1 $\beta$ and IL- 8 were observed to increase from 3 to $8 \mathrm{~h}$ after the intervention; however, the statistical analysis revealed that the only significant change from baseline levels was the increase of IL- 8 at $8 \mathrm{~h}$.

\section{Discussion}

Polyphenols are ubiquitously found in plants and comprise a major part of a daily human diet. Over the past 20 years, significant data have emerged with regard to the potential beneficial effects of several classes of phenolic compounds against a number of chronic diseases. In addition, a reasonable understanding has been gained of the bioavailability of many polyphenol classes, and this will be important for understanding the mechanisms by which they exert such benefits in vivo. The interest in marine sources of phenolic compounds is recent, and knowledge on phlorotannin bioavailability is still lacking. The analysis of phlorotannins is challenging because of the high range of molecular weight present, and their characterisation is complicated further by the lack of commercially available standards.

Chromatography techniques coupled to diode array and MS detection have been applied to the analysis of phlorotannins, and the advantages/disadvantages of their use are described by Steevensz et al. ${ }^{(34)}$. RP-HPLC is a separation mode that is commonly used for polyphenol separation; however, the very high polarity of phlorotannins would cause them to elute with little or no retention because of the lack of interaction with the nonpolar stationary phase ${ }^{(34,48)}$. NP-HPLC is more advantageous for retaining compounds with very high polarity, and the NP-HPLC methodology developed by Koivikko was more suitable than RP-HPLC for the separation of phlorotannins from the brown algae Fucus vesiculosus ${ }^{(34,48)}$. Thus, we initially analysed the phlorotannins in our SPE by NP-HPLC using a method adapted from Koivikko et al. ${ }^{(8)}$. As expected, the SPE comprised a wide range of molecular weights (20-70 min), with abundance of very HMW phlorotannins eluting at later retention time (50-70 min) in our normal-phase method. MS detection can provide higher sensitivity and be advantageous to identify specific phlorotannins in the extract without commercially available standards; however, NP-HPLC solvents such as 
(a)
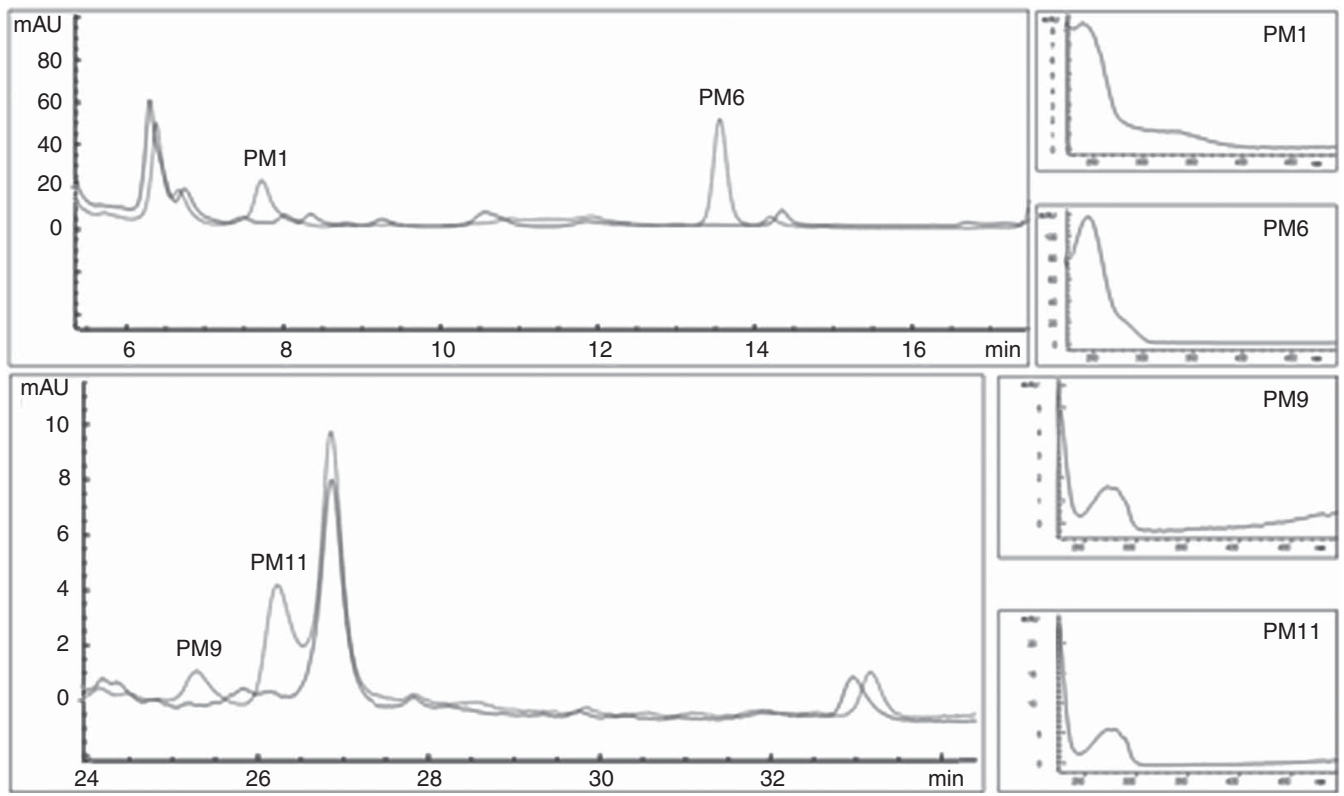

(b)

RP-HPLC analysis of seaweed metabolites in plasma samples

\begin{tabular}{|c|c|c|c|c|c|c|c|}
\hline $\mathrm{RT}$ (min) & Metabolite & $\operatorname{Mean}(\mu \mathrm{g} / \mathrm{ml})$ & SD & $n$ volunteers & Time (h) & Enz- & Enz+ \\
\hline 6.5 & PM1 & $2 \cdot 16$ & $2 \cdot 01$ & 2 & $4 ; 24$ & No & Yes \\
\hline $7 \cdot 7$ & PM2 & 0.95 & & 1 & 24 & Yes & No \\
\hline $9 \cdot 2$ & PM3 & 0.07 & & 1 & 8 & Yes & No \\
\hline $9 \cdot 3$ & PM4 & 0.07 & 0.03 & 2 & $2 ; 3$ & Yes & No \\
\hline $9 \cdot 4$ & PM5 & 0.05 & 0.03 & 7 & $3 ; 4 ; 6 ; 8 ; 24$ & Yes & No \\
\hline $13 \cdot 5$ & PM6 & $2 \cdot 82$ & $1 \cdot 85$ & 2 & 24 & Yes & Yes \\
\hline $20 \cdot 4$ & PM7 & 0.03 & & 1 & 3 & No & Yes \\
\hline $23 \cdot 2$ & PM8 & 0.03 & & 1 & 24 & No & Yes \\
\hline $25 \cdot 2$ & PM9 & 0.06 & 0.01 & 2 & $8 ; 24$ & Yes & No \\
\hline $25 \cdot 5$ & PM10 & 0.01 & 0.00 & 2 & 24 & Yes & Yes \\
\hline $26 \cdot 2$ & PM11 & 0.22 & & 1 & 24 & Yes & No \\
\hline $30 \cdot 3$ & PM12 & 0.41 & & 1 & 24 & Yes & No \\
\hline $32 \cdot 7$ & PM13 & 0.05 & 0.04 & 3 & 24 & Yes & Yes \\
\hline $33 \cdot 2$ & PM14 & 0.04 & & 1 & 4 & Yes & No \\
\hline $36 \cdot 1$ & PM15 & 0.01 & & 1 & 2 & Yes & No \\
\hline $38 \cdot 0$ & PM16 & 0.02 & 0.01 & 3 & $2 ; 3$ & Yes & No \\
\hline $42 \cdot 4$ & PM17 & 0.04 & & 1 & 4 & Yes & No \\
\hline $44 \cdot 7$ & PM18 & 0.03 & 0.03 & 5 & $6 ; 24$ & Yes & Yes \\
\hline
\end{tabular}

Fig. 5. HPLC analysis of plasma samples for seaweed metabolites. (a) HPLC chromatograms $(268 \mathrm{~nm})$ and UV spectra showing examples of metabolites in plasma. (b) Summary of seaweed metabolites present in plasma samples. PM, plasma metabolite; RP-HPLC, reverse-phase HPLC.

dichloromethane are not suitable for MS analysis ${ }^{(52)}$, because they would result in poor ionisation and therefore significantly reduce sensitivity ${ }^{(51,53)}$.

Nwosu et al. ${ }^{(29)}$ characterised a phlorotannin extract from A. nodosum by RP-HPLC using a C18 column: the bound sample eluted in a unresolved set of peaks, and with MS detection in negative ion mode they were able to assign the detected $m / z$ spectra to a series of phlorotannin structures ${ }^{(29)}$. Ferreres et al. ${ }^{(50)}$ identified twenty-two different phlorotannins belonging to the eckol and fucophloroethol groups in four seaweed species belonging to the order Fucales (genus Cystoseira and Fucus), with RP-HPLC separation combined with DAD-ESI-multiple-stage MS detection ${ }^{(50)}$. By using an equivalent RP-HPLC separation method coupled to ESI-MS analysis in negative ion mode, we were able to identify some phlorotannin oligomers such as hydroxytrifuhalol A, tetrafucol, fucodiphlorethol, C-O-C dimmer of phloroglucinol, 7-hydroxyeckol, diphloretol and difucol. The fragmentation patterns of the oligomers 
(a)
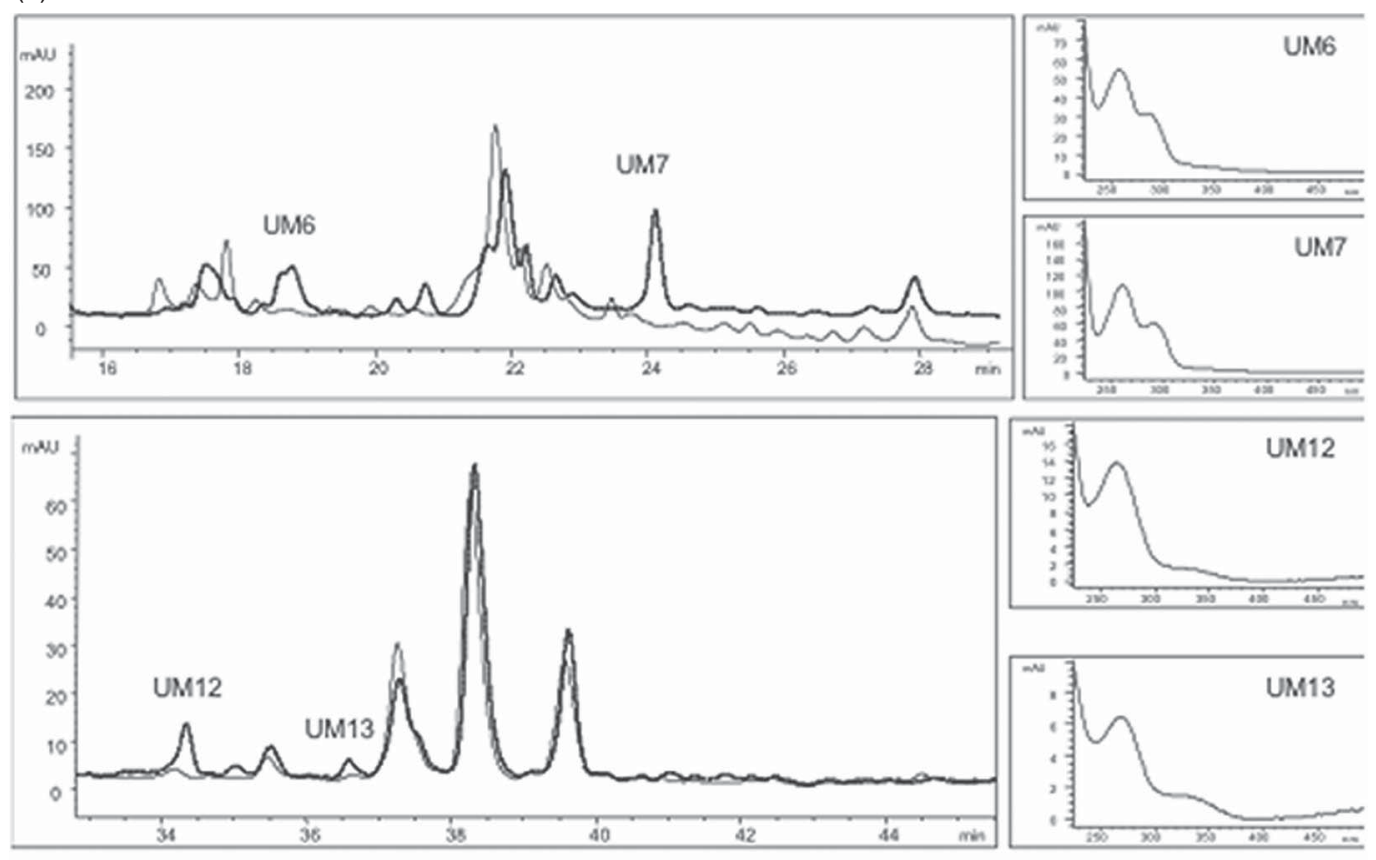

(b)

RP-HPLC analysis of seaweed metabolites in urine samples

\begin{tabular}{|c|c|c|c|c|c|c|c|}
\hline RT (min) & Metabolite & Mean (mg) & SD & $n$ volunteers & Time (h) & Enz- & Enz+ \\
\hline $7 \cdot 9$ & UM1 & $15 \cdot 25$ & $1 \cdot 39$ & 2 & $0-8 ; 8-24$ & Yes & Yes \\
\hline $10 \cdot 4$ & UM2 & $10 \cdot 77$ & $4 \cdot 29$ & 7 & $8-24$ & Yes & Yes \\
\hline $14 \cdot 2$ & UM3 & $16 \cdot 66$ & 0.89 & 2 & $8-24$ & Yes & Yes \\
\hline $16 \cdot 7$ & UM4 & $2 \cdot 94$ & $1 \cdot 82$ & 3 & $8-24$ & Yes & Yes \\
\hline $17 \cdot 7$ & UM5 & 0.67 & & 1 & 8-24 & Yes & No \\
\hline $18 \cdot 7$ & UM6 & $5 \cdot 58$ & $0 \cdot 17$ & 2 & $8-24$ & Yes & Yes \\
\hline $24 \cdot 1$ & UM7 & $9 \cdot 85$ & & 1 & $8-24$ & No & Yes \\
\hline $29 \cdot 6$ & UM8 & 0.30 & & 1 & $8-24$ & Yes & No \\
\hline $30 \cdot 8$ & UM9 & 0.51 & & 1 & $8-24$ & Yes & No \\
\hline $33 \cdot 0$ & UM10 & $1 \cdot 26$ & & 1 & $8-24$ & No & Yes \\
\hline $33 \cdot 1$ & UM11 & $1 \cdot 28$ & 0.09 & 2 & $8-24$ & Yes & Yes \\
\hline $34 \cdot 3$ & UM12 & 0.63 & $0 \cdot 15$ & 2 & $8-24$ & Yes & Yes \\
\hline $36 \cdot 5$ & UM13 & $0 \cdot 61$ & 0.55 & 6 & $8-24$ & Yes & Yes \\
\hline $41 \cdot 8$ & UM14 & 1.45 & $0 \cdot 86$ & 8 & $0-8 ; \quad 8-24$ & No & Yes \\
\hline $43 \cdot 7$ & UM15 & 0.25 & & 1 & $8-24$ & No & Yes \\
\hline $45 \cdot 5$ & UM16 & 0.69 & & 1 & $8-24$ & Yes & No \\
\hline $46 \cdot 1$ & UM17 & 0.25 & & 1 & $8-24$ & No & Yes \\
\hline $46 \cdot 7$ & UM18 & $0 \cdot 70$ & & 1 & $8-24$ & No & Yes \\
\hline
\end{tabular}

Fig. 6. HPLC analysis of urine samples for seaweed metabolites. (a) HPLC chromatograms $(268 \mathrm{~nm})$ and UV spectra showing examples of metabolites in urine. (b) Summary of seaweed metabolites present in urine samples. UM, urine metabolite; RP-HPLC, reverse-phase HPLC.

that we identified are in agreement with some recently published data in the field ${ }^{(49,50)}$. Recently, Steevensz et $a l^{(34)}$ characterised the phlorotannins of five brown algae species by ultrahigh-pressure liquid chromatography operating in 'mixedmode' (hydrophilic interaction liquid chromatography mode) combined with high-resolution MS. The methodology proposed 
(a)
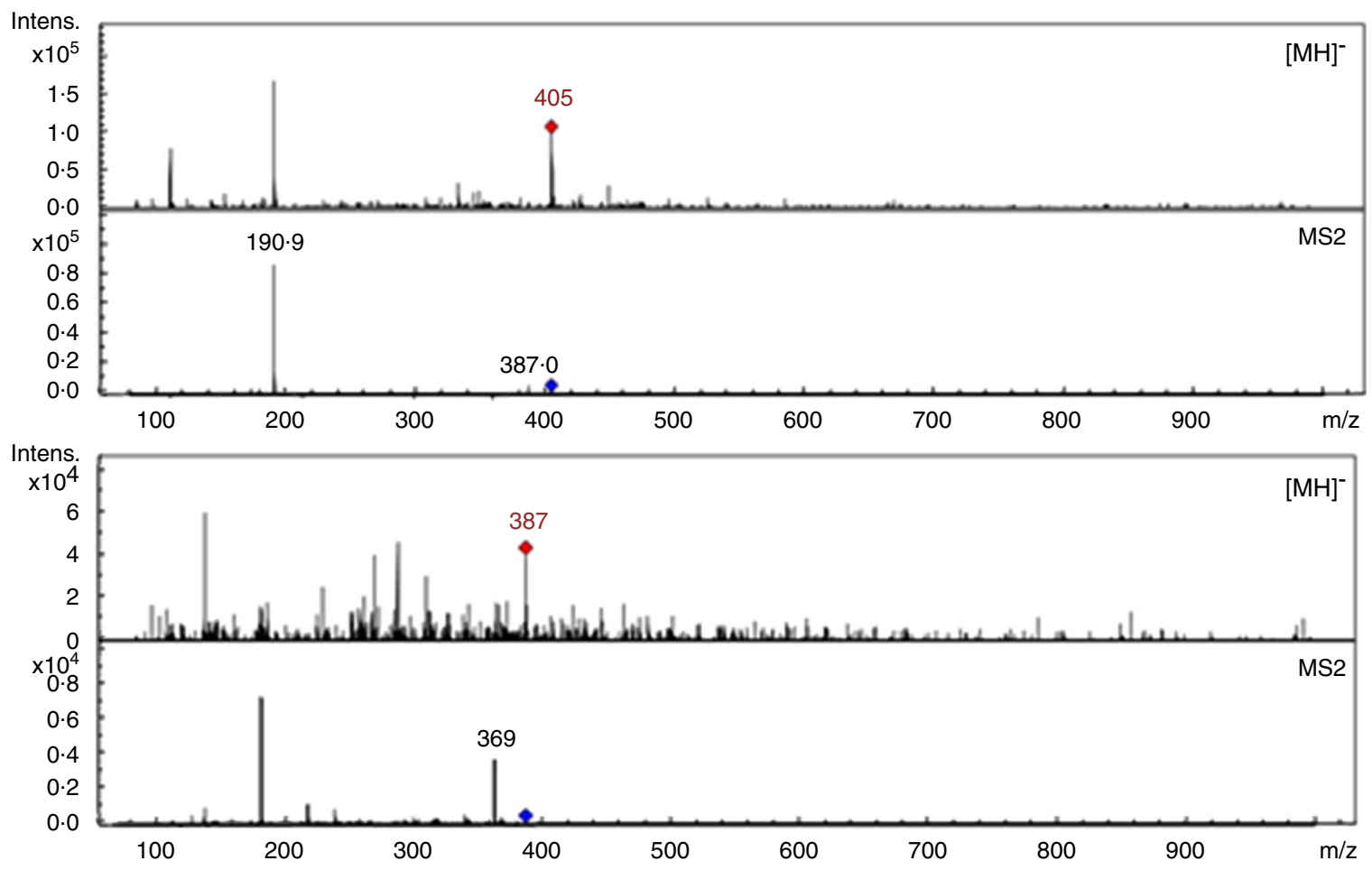

Intens.

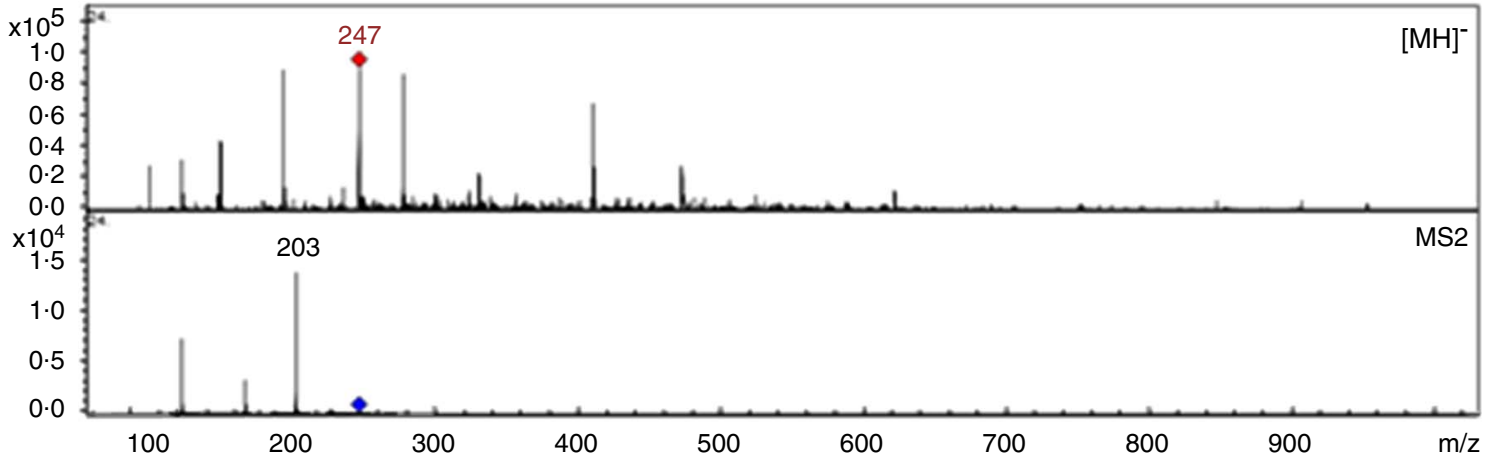

(b)

LC-MS analysis in negative ion mode of the urine samples

\begin{tabular}{|c|c|c|c|c|}
\hline \multirow{2}{*}{$\begin{array}{c}\begin{array}{c}\mathrm{RT} \\
(\mathrm{min})\end{array} \\
2 \cdot 20\end{array}$} & \multirow{2}{*}{$\begin{array}{r}{[\mathrm{M}-\mathrm{H}]-} \\
(\mathrm{m} / \mathrm{z})\end{array}$} & \multicolumn{2}{|c|}{$\begin{array}{l}\text { MS2 } \\
(\mathrm{m} / \mathrm{z})\end{array}$} & \multirow{2}{*}{$\begin{array}{c}\text { Tentative identification } \\
\text { Hydroxytrifuhalol A }\end{array}$} \\
\hline & & 387 & 191 & \\
\hline $4 \cdot 10$ & 387 & 369 & & 7-Hydroxyeckol \\
\hline $10 \cdot 40$ & 247 & 203 & & C-O-C dimer of phloroglucinol ,UMA3 \\
\hline $1 \cdot 70$ & 289 & 221 & 175 & Unknown, DM4 \\
\hline $1 \cdot 85$ & 377 & 341 & 161 & Uknown, DM7 \\
\hline
\end{tabular}

Fig. 7. Liquid chromatography (LC)-MS analysis in negative ion mode of urine samples. (a) LC-MS spectra and fragmentation of phlorotannins found in urine samples. (b) Summary of LC-MS analysis of the urine samples. DM, digestion metabolite.

by this research group proved to be accurate for profiling phlorotannins based on their degree of polymerisation, and it was demonstrated to be an effective separation mode for lowmolecular-weight phlorotannins, up to $6 \mathrm{kDa}^{(34)}$.
Phlorotannin characterisation is a challenging and complex task, which is complicated by the lack of commercially available standard compounds; thus, chromatography separation coupled to MS detection can help to elucidate phlorotannin 


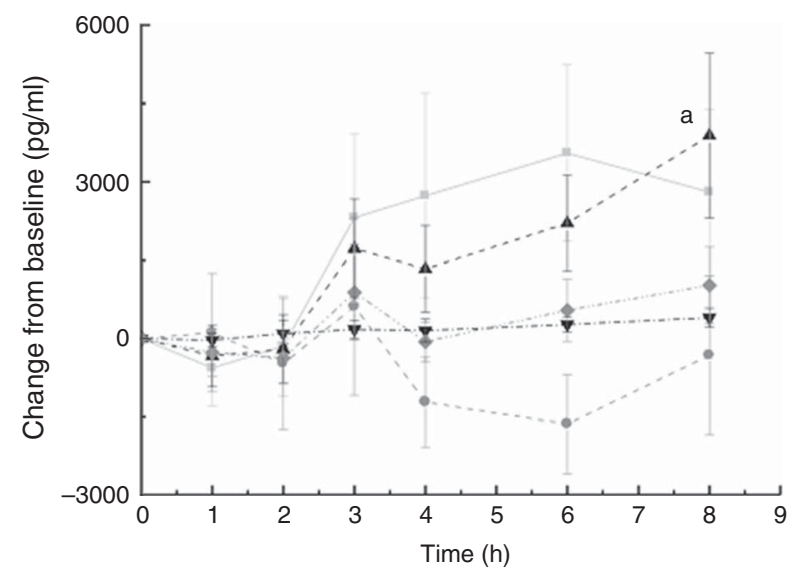

Fig. 8. Cytokine production by whole blood cultures in cultured blood collected at various time points $(0,1,2,3,4,6$ and $8 \mathrm{~h})$ during the intervention study (lipopolysaccharide treated - unstimulated controls). ${ }^{\text {a }} P<0.05 \mathrm{v}$. baseline.

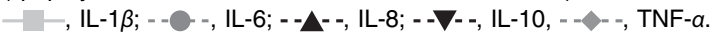

complexity, and its application to the analysis of clinical samples from feeding trials, as well as the use of simplified in vitro digestion systems, can help elucidate their beneficial health properties and the bioactive circulating forms. The SPE was subjected to in vitro-simulated GI digestion and fermentation, followed by dialysis to simulate as close as possible their absorption and biotransformation. The obtained materials were analysed by LC-MS for a comparative characterisation of the phlorotannin metabolites. LC-MA analysis of the digested and fermented SPE has indicated the presence of some oligomeric phlorotannins that are also present in the undigested SPE (hydroxytrifuhalol A, diphloretol/difucol, 7-hydroxyeckol, $\mathrm{C}-\mathrm{O}-\mathrm{C}$ dimer of phloroglucinol), in addition to a range of newly formed metabolites (DM1 to DM7). In vitro conditions are indeed a great tool, allowing a simpler and more convenient analysis, and our in vitro system predicted the formation of metabolites subsequently identified in urine.

Intervention studies have investigated the fate of polyphenols from land plants in the human body by measuring plasma concentrations and/or urinary excretion following intake from a food source. Many studies performed to investigate polyphenol bioavailability are based on the measurement of their excretion in urine and plasma by extraction, concentration and chromatographic separation/analysis, and focused on the detection of polyphenols and their metabolites in samples subjected or not to enzymatic treatment to release conjugate moieties such as glucuronic acid and sulphate groups ${ }^{(46,54)}$. For example, after ingestion of a polyphenol-rich meal, levels of phenolic compounds and conjugated metabolites can increase rapidly, achieving a peak concentration at approximately $1-2 \mathrm{~h}$ in plasma and urine, indicating small intestinal absorption, or peak at later time points ( $4-8 \mathrm{~h}$ in plasma, $8-24 \mathrm{~h}$ in urine), indicating large intestinal metabolism and subsequent absorption ${ }^{(55)}$. In our study, the majority of phlorotannin metabolites were found in samples collected at late time points $(6-24 \mathrm{~h})$, indicating limited small intestinal absorption followed by gut microbial metabolism of the HMW phlorotannins in the large intestine.

In the upper GI tract, dietary polyphenols act as substrates for a number of enzymes, and they are subjected to extensive metabolism by glucosidase enzymes, phase I enzymes (hydrolysing and oxidising), such as cytochrome P450, and phase II enzymes (conjugating and detoxifying) found both in the small intestine and the liver ${ }^{(56)}$. In particular, there is strong evidence for the extensive phase II metabolism (by UDPglucuronosyltransferases, sulphotransferases) to yield glucuronides and sulphate derivatives. Indeed, there is evidence of efficient glucuronidation and sulphation of all classes of polyphenols to differing extents ${ }^{(56)}$. Indeed, our results indicate that phlorotannin intake results in the formation of phase II conjugate metabolites (glucuronides, sulphates).

Further transformations can occur in the colon, in which the enzymes of the gut microbiota act to breakdown complex polyphenolic structures to smaller units, which may also be absorbed and further metabolised in the liver. Bacterial enzymes may catalyse many reactions including hydrolysis, dehydroxylation, demethylation, ring cleavage and decarboxylation, as well as rapid deconjugation ${ }^{(57)}$.

As predicted by the HMW range of phlorotannins in our SPE, high colonic metabolism seems to have occurred, following fermentation of HMW phlorotannins in the large intestine. By LC-MS analysis, we were able, for the first time, to confirm the presence of some phlorotannin oligomers in urine, more specifically hydroxytrifuhalol A, 7-hydroxyeckol and the C-O-C dimer of phloroglucinol. Interestingly, two of the urine metabolites $(\mathrm{m} / z 289$ and 377) were present in the in vitrodigested samples (DM4 and DM7).

There were substantial differences between volunteers in the pattern of phlorotannin metabolites. Such inter-individual differences have been observed for other polyphenols and have been attributed to differences in gut microbiota composition and the expression of metabolising enzymes ${ }^{(3,55)}$.

A secondary aim of our work has been to determine whether the SPE could modulate inflammatory events in the blood, following the absorption of phlorotannin metabolites and because of their presence in the circulation.

Polyphenols can exert numerous antioxidant and nonantioxidant functions of relevance in chronic disease development, and many of them have an important inflammatory component ${ }^{(53)}$. In the present study, we observed an altered ex vivo production of IL-8, a low-molecular-weight cytokine produced by mononuclear phagocytes and other cell types, with significant increased levels of the cytokine after $8 \mathrm{~h}$ compared with baseline.

IL-8 is an important inflammatory factor of the cysteineintervening amino acid-cysteine (CXC) chemokine family, involved in the amplification of inflammatory signals ${ }^{(59)}$. IL-8 secretion is induced by TNF- $\alpha$ through a transcriptional mechanism primarily regulated by $\mathrm{NF}-\kappa \mathrm{B})^{(60)}$. Redox signalling mechanisms are known to play a role in the regulation of such events, with reactive oxygen species being able to promote IL-8 production and secretion ${ }^{(18,23)}$, whereas oxygen radical scavengers are proven to inhibit IL-8 production in LPSstimulated human whole blood ${ }^{(22)}$. Dietary polyphenols such as catechins ${ }^{(33)}$ and curcumin ${ }^{(28)}$ have also been shown to specifically interfere with IL-8 gene expression through inhibition of $\mathrm{NF}-\kappa \mathrm{B}$ activation ${ }^{(61)}$. In consequence, we would have expected circulating seaweed polyphenol metabolites to 
potentially be able to inhibit IL- 8 secretion. Our results have given a preliminary indication that the cytokine IL- 8 is a possible target for phlorotannin metabolites. However, a significant increase in IL- 8 levels at $8 \mathrm{~h}$ after the intervention was observed, in parallel with the presence of phlorotannin metabolites in plasma and urine samples. A recent study from our group investigated the influence of a polyphenol-rich intervention on inflammation as primary outcome. A randomised, double-blind, placebo-controlled, cross-over acute intervention was conducted, and cytokine levels (IL-8) were measured with the same ex vivo experimental protocol. The results showed a timedependent increase in IL-8 release compared with baseline, in accordance with our findings. Thus, the postprandial ex vivo IL-8 production was significantly attenuated by the intervention compared with the control, with a parallel appearance in the circulation of polyphenol metabolites. Our trial was a singlegroup interventional study primarily designed to investigate the bioavailability of seaweed phlorotannins: however, on the basis of this preliminary indication on their anti-inflammatory potential, not sufficient to draw any conclusion, a chronic placebo-controlled intervention has been conducted to investigate the anti-inflammatory effect in deeper detail.

The main limitations of this study arise from the phlorotannin complexity and lack of commercially available analytical standards, potentially leading to possible quantification inaccuracy as phloroglucinol equivalents. The lack of analytical standards also implies a limited capability for method development, especially for the analysis of plasma, urine and digested materials. In future, the availability of standards could allow a higher degree of method optimisation and the development of specific solidphase ion procedures for sample clean-up and concentration.

The development of more recently explored analytical applications to phlorotannins, such as hydrophilic interaction liquid chromatography ${ }^{(32)}$ and $\mathrm{NMR}^{(35)}$, could facilitate the development of more suitable protocols that could lead to full identification of metabolites and improvements in phlorotannin metabolite quantification. In addition, the bioacessibility of polyphenols in the form of a capsule/extract might differ greatly from the bioacessibility of the same compounds in a food matrix ${ }^{(62)}$. Future work will be needed to determine the potential effects on bioavailability of different food matrices and also any effects of cooking and/or processing.

Nevertheless, the present work has for the first time started to shed light on the role of colonic biotransformation on phlorotannin bioavailability, and its implication for their health benefits in vivo. Our results provide a basis for further investigating the seaweed-derived bioactive components in the body after ingestion; this information is necessary to understand their mechanism of action in vivo.

\section{Acknowledgements}

This work is part of the SWAFAX project (no. 262519), funded by the European Commission under the Capacities Programme (FP7)

We would like to thank all the SWAFAX project partners for their contribution, including Marigot, Hebridean Seaweed, Mesosystem S.L., Cybercolloids and the University of Ulster.
G. C., J. P. E. S., P. Y. and I. R. designed the research. G. C. organised and coordinated all parts of the clinical trial and analytical work. S. H. overviewed the preparation of seaweed materials, extracts and capsules. P. A. aided running the clinical trial. Y. J. aided with the analysis of metabolites in plasma and urines. G. C. analysed and summarised all the data. G. C. drafted the manuscript, C. G., I. R., S. H. and P. Y. revised the manuscript. I. R. had primary responsibility for final content.

The authors declare that there are no conflicts of interest.

\section{References}

1. MacArtain P, Gill CI, Brooks M, et al. (2007) Nutritional value of edible seaweeds. Nutr Rev 65, 535-543.

2. Park EJ \& Pezzuto JM (2013) Antioxidant marine products in cancer chemoprevention. Antioxid Redox Signal 19, 115-138.

3. Brown EM, Allsopp PJ, Magee PJ, et al. (2014) Seaweed and human health. Nutr Rev 72, 205-216.

4. Arasaki S \& Arasaki T (1983) Low Calorie, High Nutrition Vegetables from the Sea to Help You Look and Feel Better, 1st ed. Tokyo and New York, NY: Japan Publications; Distributor, US, Kodansha International/USA, through Harper \& Row.

5. Zava TT \& Zava DT (2011) Assessment of Japanese iodine intake based on seaweed consumption in Japan: a literaturebased analysis. Thyroid Res $\mathbf{4}, 14$.

6. Guiry MD \& Blunden G (1991) Seaweed Resources in Europe: Uses and Potential. Chichester and New York, NY: Wiley.

7. Matsumura Y (2001) Nutrition trends in Japan. Asia Pac J Clin Nutr 10, Suppl, S40-S47.

8. Koivikko R, Eranen JK, Loponen J, et al. (2008) Variation of phlorotannins among three populations of Fucus vesiculosus as revealed by HPLC and colorimetric quantification. $J$ Chem Ecol 34, 57-64.

9. Dutot M, Fagon R, Hemon M, et al. (2012) Antioxidant, anti-inflammatory, and anti-senescence activities of a phlorotannin-rich natural extract from brown seaweed Ascophyllum nodosum. Appl Biochem Biotechnol 167, 2234-2240.

10. Wijesinghe WA \& Jeon YJ (2012) Exploiting biological activities of brown seaweed Ecklonia cava for potential industrial applications: a review. Int J Food Sci Nutr 63, 225-235.

11. Ragan MA \& Craigie JS (1976) Physodes and the phenolic compounds of brown algae. Isolation and characterization of phloroglucinol polymers from Fucus vesiculosus (L.). Can J Biochem 54, 66-73.

12. Glombitza KW, Hauperich S \& Keusgen M (1997) Phlorotannins from the brown algae Cystophora torulosa and Sargassum spinuligerum. Nat Toxins 5, 58-63.

13. Glombitza KW \& Schmidt A (1999) Nonhalogenated and halogenated phlorotannins from the brown alga carpophyllum angustifolium. J Nat Prod 62, 1238-1240.

14. Glombitza KW \& Vogels HP (1985) Antibiotics from algae. XXXV. Phlorotannins from Ecklonia maxima1. Planta Med $\mathbf{5 1}$, 308-312.

15. Glombitza KW \& Zieprath G (1989) Phlorotannins from the brown alga Analipus japonicus1. Planta Med 55, 171-175.

16. Pal Singh I \& Bharate SB (2006) Phloroglucinol compounds of natural origin. Nat Prod Rep 23, 558-591.

17. Kim SK \& Himaya SW (2011) Medicinal effects of phlorotannins from marine brown algae. Adv Food Nutr Res 64, 97-109.

18. Tsujimoto M, Yokota S, Vilcek J, et al. (1986) Tumor necrosis factor provokes superoxide anion generation from neutrophils. Biochem Biophys Res Commun 137, 1094-1100. 
19. Kang MC, Cha SH, Wijesinghe WA, et al. (2013) Protective effect of marine algae phlorotannins against AAPH-induced oxidative stress in zebrafish embryo. Food Chem 138, 950-955.

20. Kim AR, Shin TS, Lee MS, et al. (2009) Isolation and identification of phlorotannins from Ecklonia stolonifera with antioxidant and anti-inflammatory properties. I Agric Food Chem 57, 3483-3489.

21. Kim SM, Kang K, Jeon JS, et al. (2011) Isolation of phlorotannins from Eisenia bicyclis and their hepatoprotective effect against oxidative stress induced by tert-butyl hyperoxide. Appl Biochem Biotechnol 165, 1296-1307.

22. DeForge LE, Fantone JC, Kenney JS, et al. (1992) Oxygen radical scavengers selectively inhibit interleukin 8 production in human whole blood. J Clin Invest 90, 2123-2129.

23. McCord JM (1987) Oxygen-derived radicals: a link between reperfusion injury and inflammation. Fed Proc $\mathbf{4 6}$ 2402-2406.

24. Queguineur B, Goya L, Ramos S, et al. (2013) Effect of phlorotannin-rich extracts of Ascophyllum nodosum and Himanthalia elongata (Phaeophyceae) on cellular oxidative markers in human HepG2 cells. J Appl Phycol 25, 1-11.

25. Jung HA, Jin SE, Ahn BR, et al. (2013) Anti-inflammatory activity of edible brown alga Eisenia bicyclis and its constituents fucosterol and phlorotannins in LPS-stimulated RAW264.7 macrophages. Food Chem Toxicol 59, 199-206.

26. Kim TH, Ku SK, Lee T, et al. (2012) Vascular barrier protective effects of phlorotannins on HMGB1-mediated proinflammatory responses in vitro and in vivo. Food Chem Toxicol 50, 2188-2195.

27. Eom $\mathrm{SH}$, Kim $\mathrm{DH}$, Lee $\mathrm{SH}$, et al. (2013) In vitro antibacterial activity and synergistic antibiotic effects of phlorotannins isolated from Eisenia bicyclis against methicillin-resistant Staphylococcus aureus. Phytother Res 27, 1260-1264.

28. Biswas SK, McClure D, Jimenez LA, et al. (2005) Curcumin induces glutathione biosynthesis and inhibits NF-kappaB activation and interleukin-8 release in alveolar epithelial cells: mechanism of free radical scavenging activity. Antioxid Redox Signal 7, 32-41.

29. Nwosu F, Morris J, Lund VA, et al. (2010) Anti-proliferative and potential anti-diabetic effects of phenolic-rich extracts from edible marine algae. Food Chem 126, 1006-1012.

30. Ryu B, Li Y, Qian ZJ, et al. (2009) Differentiation of human osteosarcoma cells by isolated phlorotannins is subtly linked to COX-2, iNOS, MMPs, and MAPK signaling: implication for chronic articular disease. Chem Biol Interact 179, 192-201.

31. Yoon NY, Eom TK, Kim MM, et al. (2009) Inhibitory effect of phlorotannins isolated from Ecklonia cava on mushroom tyrosinase activity and melanin formation in mouse B16F10 melanoma cells. J Agric Food Chem 57, 4124-4129.

32. Melanson JE \& MacKinnon SL (2015) Characterization of phlorotannins from brown algae by LC-HRMS. Methods Mol Biol 1308, 253-266.

33. Wheeler DS, Catravas JD, Odoms K, et al. (2004) Epigallocatechin-3-gallate, a green tea-derived polyphenol, inhibits IL-1 beta-dependent proinflammatory signal transduction in cultured respiratory epithelial cells. J Nutr 134, 1039-1044.

34. Steevensz AJ, Mackinnon SL, Hankinson R, et al. (2012) Profiling phlorotannins in brown macroalgae by liquid chromatography-high resolution mass spectrometry. Phytochem Anal 23, 547-553.

35. Jegou C, Kervarec N, Cerantola S, et al. (2015) NMR use to quantify phlorotannins: the case of Cystoseira tamariscifolia, a phloroglucinol-producing brown macroalga in Brittany (France). Talanta 135, 1-6.

36. Wijesinghe WA \& Jeon YJ (2012) Exploiting biological activities of brown seaweed Ecklonia cava for potential industrial applications: a review. Int J Food Sci Nutr 63, 225-235.

37. Corona G, Vauzour D, Amini A, et al. (2014) The impact of gastrointestinal modifications, blood brain barrier transport, and intracellular metabolism on polyphenol bioavailability: an overview. In Polyphenols in Human Health and Disease, pp. 591-604 [RR Watson, VR Preedy and S Zibadi, editors]. San Diego, CA: Elsevier-Academic Press.

38. Vauzour D, Rodriguez-Mateos A, Corona G, et al. (2010) Polyphenols and human health: prevention of disease and mechanisms of action. Nutrients 2, 1106-1131.

39. Manach C, Scalbert A, Morand C, et al. (2004) Polyphenols: food sources and bioavailability. Am J Clin Nutr 79, 727-747.

40. Scalbert A, Morand C, Manach C, et al. (2002) Absorption and metabolism of polyphenols in the gut and impact on health. Biomed Pharmacother 56, 276-282.

41. Simmons-Boyce JL, Purcell SL, Nelson CM, et al. (2009) Dietary Ascophyllum nodosum increases urinary excretion of tricarboxylic acid cycle intermediates in male SpragueDawley rats. J Nutr 139, 1487-1494.

42. Singleton VL (1985) Citation classic-colorimetry of total phenolics with phosphomolybdic-phosphotungstic acid reagents. CC/Agr Biol Environ 16, 18-18.

43. Mills DJ, Tuohy KM, Booth J, et al. (2008) Dietary glycated protein modulates the colonic microbiota towards a more detrimental composition in ulcerative colitis patients and nonulcerative colitis subjects. $J$ Appl Microbiol 105, 706-714.

44. McDougall GJ, Dobson P, Smith P, et al. (2005) Assessing potential bioavailability of raspberry anthocyanins using an in vitro digestion system. J Agric Food Chem 53, 5896-5904.

45. Tzounis X, Vulevic J, Kuhnle GG, et al. (2008) Flavanol monomer-induced changes to the human faecal microflora. Br J Nutr 99, 782-792.

46. Ottaviani JI, Momma TY, Heiss C, et al. (2011) The stereochemical configuration of flavanols influences the level and metabolism of flavanols in humans and their biological activity in vivo. Free Radic Biol Med 50, 237-244.

47. Corona G, Tzounis X, Assunta Dessi M, et al. (2006) The fate of olive oil polyphenols in the gastrointestinal tract: implications of gastric and colonic microflora-dependent biotransformation. Free Radic Res 40, 647-658.

48. Pinto J, Paiva-Martins F, Corona G, et al. (2011) Absorption and metabolism of olive oil secoiridoids in the small intestine. Br J Nutr 105, 1607-1618.

49. Wang $\mathrm{T}$, Jonsdottir $\mathrm{R}$, Liu $\mathrm{H}$, et al. (2012) Antioxidant capacities of phlorotannins extracted from the brown algae Fucus vesiculosus. J Agric Food Chem 60, 5874-5883.

50. Ferreres F, Lopes G, Gil-Izquierdo A, et al. (2012) Phlorotannin extracts from fucales characterized by HPLC-DADESI-MSn: approaches to hyaluronidase inhibitory capacity and antioxidant properties. Mar Drugs 10, 2766-2781.

51. Koivikko R, Loponen J, Pihlaja K, et al. (2007) Highperformance liquid chromatographic analysis of phlorotannins from the brown alga Fucus vesiculosus. Phytochem Anal 18, 326-332.

52. Kazakevich Y \& LoBrutto R (2007) HPLC for Pharmaceutical Scientists. Hoboken, NJ: Wiley-Interscience.

53. Yanagida A, Murao H, Ohnishi-Kameyama M, et al. (2007) Retention behavior of oligomeric proanthocyanidins in hydrophilic interaction chromatography. J Chromatogr A 1143, 153-161.

54. Miro-Casas E, Covas MI, Farre M, et al. (2003) Hydroxytyrosol disposition in humans. Clin Chem 49, 945-952. 
55. Rodriguez-Mateos A, Rendeiro C, Bergillos-Meca $\mathrm{T}$, et al (2013) Intake and time dependence of blueberry flavonoidinduced improvements in vascular function: a randomized, controlled, double-blind, crossover intervention study with mechanistic insights into biological activity. Am J Clin Nutr $\mathbf{9 8}$, 1179-1191.

56. Manach C, Williamson G, Morand C, et al. (2005) Bioavailability and bioefficacy of polyphenols in humans. I. Review of 97 bioavailability studies. Am J Clin Nutr 81, 230S-242S.

57. Selma MV, Espin JC \& Tomas-Barberan FA (2009) Interaction between phenolics and gut microbiota: role in human health. J Agric Food Chem 57, 6485-6501.
58. Suarez M, Valls RM, Romero MP, et al. (2011) Bioavailability of phenols from a phenol-enriched olive oil. Br J Nutr 106, 1691-1701.

59. Harada A, Sekido N, Akahoshi T, et al. (1994) Essential involvement of interleukin-8 (IL-8) in acute inflammation. J Leukoc Biol 56, 559-564.

60. Fujishima S, Hoffman AR, Vu T, et al. (1993) Regulation of neutrophil interleukin 8 gene expression and protein secretion by LPS, TNF-alpha, and IL-1 beta. J Cell Physiol 154, 478-485.

61. Rahman I, Biswas SK \& Kirkham PA (2006) Regulation of inflammation and redox signaling by dietary polyphenols. Biochem Pharmacol 72, 1439-1452.

62. Bohn T (2014) Dietary factors affecting polyphenol bioavailability. Nutr Rev 72, 429-452. 